\title{
The renin-angiotensin-aldosterone system blockade and arterial stiffness in renal transplant recipients - a cross-sectional prospective observational clinical study
}

\author{
Źbigniew Heleniak1 ${ }^{\otimes}$, Sarah Illersperger², Susanne Brakemeier², Paul Bach³, Alicja Dębska- \\ Ślizień1, Klemens Budde² and Fabian Halleck²
}

1Department of Nephrology, Transplantology and Internal Medicine Medical University of Gdansk, Poland, Gdańsk, Poland; 2 Medizinische Klinik mit Schwerpunkt Nephrologie und Internistische Intensivmedizin Charité - Universitätsmedizin Berlin, Berlin, Germany; ${ }^{3 H u m b o l d t-U n i v e r s i t a ̈ t ~}$ zu Berlin School of Business and Economics Applied Statistics, Berlin Germany

Introduction. Arterial stiffness parameters can be used as a predictor of cardiovascular events in the general population and renal transplant recipients (RTRs). Additionally, the renin-angiotensin-aldosterone-system (RAAS) blockade mitigates arterial stiffness in the general population. There are no sufficient data concerning the role of the RAAS blockade in reducing arterial stiffness among patients after kidney transplantation. The aim of this study is to assess the influence of the above blockade on arterial stiffness in RTRs. Methods. 344 stable RTRs were enrolled in the study. 204 (59.3\%) of them received RAAS blockers (angiotensin convertase inhibitors - ACEls or angiotensin receptor blockers - ARBs): group RAAS (+), and $140(40.7 \%)$ were not treated with such agents: group RAAS (-). Results. In the RAAS (+) group, $55.9 \%$ of the patients used ARBs and $44.1 \%$ ACEls. Cardiovascular disease (coronary artery disease and/or peripheral obliterans artery disease) $(27.9 \%$ vs $14.3 \%, p<0.05)$, and heart failure $(27.4 \%$ vs $24.3 \%$, $\mathrm{p}<0.05)$ were significantly more often diagnosed in the RAAS (+) group when compared to the RAAS (-) group. Systolic blood pressure, diastolic blood pressure and all arterial stiffness parameters (baPWV, cfPWV, pulse pressure) did not differ significantly between the RAAS (+) and RAAS (-) groups. The results revealed that cardiovascular disease in patients was associated with a significant increase in both, the PWV and pulse pressure. No difference between the arterial stiffness parameters was observed in patients with a cardiovascular disease, diabetes and heart failure in the RAAS (+) and RAAS (-) groups. Moreover, beta-blockers and diuretics ameliorated the arterial stiffness parameters. Conclusions: This study showed the indication bias of the RAAS prescription, and no conclusion on the influence of RAAS on arterial stiffness can be drawn. The results indicated diuretics and beta-blockers as agents lowering the arterial stiffness in RTRs.

Key words: pulse wave velocity, kidney transplantation, hypertensive agent

Received: 14 September, 2020; revised: 25 October, 2020; accepted: 25 October, 2020; available on-line: 17 December, 2020

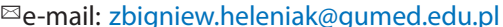

Abbreviations: ACEi, angiotensin convertase inhibitors; ARBs, angiotensin receptor blockers; baPWV, brachial-ankle pulse wave velocity; CVD, cardiovascular disease; cfPWV, carotid-femoral pulse wave velocity; RTR, renal transplant recipients; RAAS, renin-angiotensin aldosterone-system

\section{INTRODUCTION}

Kidney transplantation (KTx) is the optimal form of renal replacement therapy in patients with end-stage renal disease. During the last decades, although the transplantation procedures and immunosuppressive treatment have improved, the patients still live shorter than the general population. The most common causes of death among renal transplant recipients (RTRs) are cardiovascular diseases (Kim et al., 2015). The cardiovascular complications in RTRs are not only associated with standard risk factors, but also with non-traditional factors, such as immunosuppressive therapy, earlier dialysis therapy, proteinuria, inflammation or anemia, specific for this population. The higher value of arterial stiffness is the consequence of the risk factors, and it can lead to a cardiovascular disease (CVD) (Boutouyrie et al. 2015, Holdaas et al., 2017).

Estimation of aortic stiffness can be made by measurement of the pulse wave velocity (PWV) as a direct, noninvasive method. According to the literature, the PWV value was related to all-cause and cardiovascular mortality (Laurent et al. 2006; Laurent et al., 2001; Mattace-Raso et al., 2006). In RTRs, increased stiffness predicts the incidence of cardiovascular episodes and the deterioration of renal graft function (Barenbrock et al., 2002; Bahous et al., 2004).

Scientific data showed the potential clinical benefits of angiotensin-converting enzyme inhibitors (ACEIs) or blockers of angiotensin receptor (ARBs) in chronic kidney disease.

Silvariño and others (Silvariño et al,. 2019) presented that administration of ARBs and ACEIs is associated with a slower CKD progression and a more significant proteinuria reduction in an observational study in $\mathrm{K} T \mathrm{~T}$ patients.

Additionally, in a chronic kidney disease group, the inhibition of the renin-angiotensin-aldosterone system (RAAS) independently resulted in the reduction of hypertension and PWV (Frimodt-Møller et al., 2012).

The majority of RTRs have graft failure after transplantation. This is associated with chronic allograft nephropathy, transplant glomerulopathy, recurrent and de novo renal disease, and immunosuppressive drug toxicity. Therefore, a nephroprotection treatment should be administered to slow down the decline of the graft function. The therapy must consist of appropriate immunosuppression, the blood pressure and lipid control, and the use ACEIs or ARBs (Seron et al., 2001). 
After kidney transplantation, the above agents are used not only because of hypertension but also to reduce proteinuria and for treating post-transplant erythrocytosis (Vlahakos et al., 2003) Nowadays, there is no sufficient knowledge concerning post-transplantation ACEIs or ARBs therapy in comparison to other antihypertensive agents reducing arterial stiffness.

Therefore, we performed this study concerning the administration of RAAS blockade and its influence on aortic stiffness in RTRs.

\section{METHODS}

344 stable RTRs, transplanted between 1994 and 2018, which were treated in the outpatient unit of the Department of Nephrology Charité-Universitätsmedizin Berlin, Germany, between February and July 2018, and were enrolled in the study, and also signed a written consent. The research was approved by the Ethics Committee of Charité - Universitätsmedizin Berlin (EA 1/252/17).

Patients gave their written informed consent. The study was conducted under the Declaration of Helsinki.

The information concerning demographic data, immunosuppressive treatment, renal transplantation, cardiovascular and diabetic status was obtained from the patients' medical files. Additionally, the schedule of hypertensive treatment was analyzed, including administration of ACEIs and ARBs or aldosterone antagonists. Laboratory data, such as the levels of serum creatinine, potassium, hemoglobin, proteinuria, albuminuria and NT-proBNP (N-terminal pro-B type natriuretic peptide) was obtained from medical records.

Additionally, the arterial stiffness parameters: brachialankle and carotid-femoral pulse wave velocity (baPWV left and right, cfPWV), ankle-brachial index, pulse pressure, pulsatile stress test left and right (pulsatile stress test=heart rate $\times$ pulse pressure) and blood pressure, were assessed in each patient using the ABI system 100 (Boso Bosch and Sohn, Germany) [12].

Statistical analyses. Statistical analyses were performed using the STATISTICA 13.0 PL for Windows software package. Categorical variables are presented as absolute numbers (percentages). Continuous variables are presented as mean value \pm standard deviation (S.D.) or as the median and interquartile range (IQR) for highly skewed variables. Differences in the distribution of continuous variables were assessed using the t-tests or Mann-Whitney U-test, respectively. The Chi-squared test was used for categorical variables. Pearson correlation coefficients were calculated to assess the linear association between continuous variables. Additionally, a linear regression model was used to evaluate the relationship between a dependent variable and one or more independent variables.

The Shapiro-Wilk test was used to assess normality of the continuous variables. In all statistical tests, a $p$-value $<0.05$ was considered as statistically significant.

\section{RESULTS}

\section{Study population characteristics}

Overall, 344 patients were enrolled in the study. The study population was divided into two groups based on the use of RAAS blockade. $204(59.3 \%)$ and $140(40.7 \%)$ RTRs were qualified to the RAAS $(+)$ and RAAS (-) groups, respectively.
In the group of RAAS $(+)$, as compared to RAAS $(-)$, there were more patients with CVD (coronary artery disease and/or peripheral obliterans artery disease) $(27.9 \%$ vs $14.3 \%, p<0.05)$, heart failure $(27.4 \%$ vs $24.3 \%$, $p<0.05)$ and diabetes (20\% vs $17.1 \%$, p-ns). Moreover, there were significantly more males in the RAAS (+) group $(p<0.05)$. The mean age in the RAAS $(+)$ group was higher (54 vs 50.8 years, p-ns), as compared to RAAS (-) patients.

General characteristics of the participants are summarized in Table 1. The main causes of end-stage renal disease in the study population were glomerulonephritis, tubulointerstitial nephropathy and polycystic kidney disease. In the RAAS $(+)$ group, the average serum creatinine (1.58 vs $1.36 \mathrm{mg} / \mathrm{dl}, p<0.05)$ was higher and it resulted in lower estimated glomerular filtration rate (eGFR CKD-EPI) (47.9 vs $\left.54 \mathrm{ml} / \mathrm{min} / 1.73 \mathrm{~m}^{2}, p<0.05\right)$, as compared to the RAAS (-) group. On the other hand, proteinuria (197 vs $157 \mathrm{mg} /$ day) and albuminuria (67 vs $28 \mathrm{mg} /$ day) were significantly higher in the RAAS (+) group participants. The levels of albumin in the blood and hemoglobin were similar in both groups.

Renal replacement therapy time before transplantation was similar for both groups, but the time after transplantation was longer (95.5 vs 54.5 months, $p<0.05$ ) in the RAAS (+) group.

\section{Immunosuppressive regimen in the study population}

Calcineurin inhibitors were used in $83.1 \%$. Cyclosporine was administered in $27.5 \%$ vs $12.1 \% \quad(p<0.05)$ and tacrolimus $52.9 \%$ vs $75 \%(p<0.05)$ in the RAAS (+) and RAAS (-) groups, respectively.

There was no significant difference observed in terms of mycophenolate mofetil, mycophenolate sodium, azathioprine, mTOR inhibitors, and belatacept administration between both groups.

\section{Hypertensive regimen and erythropoietin treatment in the study population}

Calcium channel blockers were administered in $52 \%$ vs $37.1 \%(p<0.05)$ and diuretics $42.2 \%$ vs $24.3 \%(p<0.05)$ in the RAAS (+) and RAAS (-) groups, respectively.

Around $70 \%$ of RTRs received beta-blockers; the groups did not differ in that respect.

There was some difference in erythropoietin administration, $27.4 \%$ vs $15 \%(p=0.07)$ in the RAAS $(+)$ and RAAS (-) groups.

Overall, $59.3 \%$ of screened RTRs had received the RAAS blockade. $55.4 \%$ of them used ARBs, and $44.6 \%$ used ACEIs. The majority of patients treated with ARBs or ACEIs received candesartan and ramipril. The mean dose of ARBs and ACEIs was 0.25 and 0.5 of their maximal recommended doses, respectively.

According to data in the patients' medical files, the most common indications for RAAS blockade were CVD $(74 \%)$, diabetes $(63.9 \%)$, and proteinuria $>150 \mathrm{mg} / \mathrm{g}$ creatinine $(63 \%)$.

Additional details concerning comorbidities, immunosuppressive, antihypertensive and erythropoietin treatments are presented in Table 1.

\section{Blood pressure control and arterial stiffness in the study population}

In the study population, the mean value of systolic blood pressure and diastolic blood pressure on the right and left arm was $140.7 \mathrm{mmHg}, 139.6 \mathrm{mmHg}$, $85.8 \mathrm{mmHg}$, and $85.7 \mathrm{mmHg}$ (p-ns), respectively. Addi- 
Table 1. Characteristics of the study population

\begin{tabular}{|c|c|c|c|c|}
\hline & Total study population & RAAS (+) & RAAS (-) & $\begin{array}{l}\text { P } \\
\text { RAAS+ vs RAAS- }\end{array}$ \\
\hline n (\%) & 344 & $204(59.3)$ & $140(40.7)$ & $<0.05$ \\
\hline $\begin{array}{l}\text { Sex F n (\%) } \\
M n(\%)\end{array}$ & $\begin{array}{l}129(37.5) \\
215(62.5)\end{array}$ & $\begin{array}{l}70(34.3) \\
134(65.7)\end{array}$ & $\begin{array}{l}59(42.1) \\
81(57.9)\end{array}$ & ns \\
\hline Age (years) Mean value + S.D. & $52.7+13.9$ & $54.0+13.7$ & $50.8+14.1$ & ns \\
\hline BMI (kg/m2) & $25.62+4.78$ & $25.94+4.41$ & $25.16+5.25$ & ns \\
\hline $\begin{array}{l}\text { Weight (kg) } \\
\text { Median (IQR) }\end{array}$ & $\begin{array}{l}75.5 \\
(63.7-86.2)\end{array}$ & $\begin{array}{l}77.8 \\
(65.6-88.2)\end{array}$ & $\begin{array}{l}73.9 \\
(61.2-84.4)\end{array}$ & $<0.05$ \\
\hline $\begin{array}{l}\text { Diabetes mellitus } \mathrm{n}(\%) \\
\text { (any type } 1,2, \text { NODAT) }\end{array}$ & $65(18.4)$ & $41(20)$ & $24(17.1)$ & ns \\
\hline Cardiovascular disease n (\%) (CAD, POAD) & $77(22.4)$ & $57(27.9)$ & $20(14.3)$ & $<0.05$ \\
\hline Heart failure n (\%) & $90(26.2)$ & $56(27.4)$ & $34(24.3)$ & $<0.05$ \\
\hline Hypertension n (\%) & $298(86.6)$ & $183(89.7)$ & $115(82.1)$ & ns \\
\hline \multicolumn{5}{|l|}{ Reasons of ESRD and KTx: $n(\%)$} \\
\hline - primary glomerulonephritis & $186(54.1)$ & $113(55.4)$ & $73(52.1)$ & ns \\
\hline - diabetic nephropathy & $5(1.5)$ & $4(2)$ & $1(0.7)$ & ns \\
\hline - polycystic kidney disease & $56(16.3)$ & $30(14.7)$ & $26(18.6)$ & ns \\
\hline - tubulointerstitial nephritis & $70(20.3)$ & $41(20.1)$ & $29(20.7)$ & ns \\
\hline - hypertensive nephropathy & $18(5.2)$ & $13(6.4)$ & $5(3.6)$ & ns \\
\hline - unknown etiology & $10(2.9)$ & $3(1.5)$ & $7(5)$ & ns \\
\hline $\begin{array}{l}\text { Time of RRT (months) } \\
\text { before transplantation } \\
\text { Median (IQR) }\end{array}$ & $\begin{array}{l}58.5 \\
(19-97)\end{array}$ & $\begin{array}{l}50 \\
(19-90.5)\end{array}$ & $\begin{array}{l}69 \\
(21-108.5)\end{array}$ & ns \\
\hline $\begin{array}{l}\text { Time after Ktx (months) } \\
\text { Median (IQR) }\end{array}$ & $\begin{array}{l}73 \\
(28-140)\end{array}$ & $\begin{array}{l}95.5 \\
(51-171.5)\end{array}$ & $\begin{array}{l}54.5 \\
(12-91.25)\end{array}$ & $<0.05$ \\
\hline $\begin{array}{l}\text { Preemptive KTx } \\
\text { n (\%) }\end{array}$ & $46(13.4)$ & $26(12.7)$ & $20(14.3)$ & ns \\
\hline $\begin{array}{l}\text { Serum creatinine level }(\mathrm{mg} / \mathrm{dl}) \\
\text { Median }(\mathrm{IQR})\end{array}$ & $\begin{array}{l}1.47 \\
(1.19-1.92)\end{array}$ & $\begin{array}{l}1.58 \\
(1.22-2.04)\end{array}$ & $\begin{array}{l}1.36 \\
(1.16-1.81)\end{array}$ & $<0.05$ \\
\hline $\begin{array}{l}\text { eGFR CKD-EPI } \\
\text { (ml/min/1.73m2) } \\
\text { Mean value + S.D. }\end{array}$ & $50.4+19.8$ & $47.9+19.4$ & $54.0+20.0$ & $<0.05$ \\
\hline $\begin{array}{l}\text { Potassium mmol/l } \\
\text { Mean value + S.D. }\end{array}$ & $4.3+0.6$ & $4.5+0.6$ & $4.2+0.5$ & $<0.05$ \\
\hline $\begin{array}{l}\text { Hemoglobin } \mathrm{g} / \mathrm{dl} \\
\text { Mean value +S.D. }\end{array}$ & $12.7+1.7$ & $12.6+1.6$ & $12.8+2.0$ & ns \\
\hline $\begin{array}{l}\text { Albumin } g / l \\
\text { Mean value + S.D. }\end{array}$ & $43.46+3.28$ & $43.33+3.41$ & $43.64+3.09$ & ns \\
\hline $\begin{array}{l}\text { Albuminuria mg/day } \\
\text { Median (IQR) }\end{array}$ & $\begin{array}{l}1.5 \\
(9.3-145)\end{array}$ & $\begin{array}{l}67 \\
(11-236)\end{array}$ & $\begin{array}{l}28 \\
(8-75)\end{array}$ & $<0.05$ \\
\hline $\begin{array}{l}\text { Proteinuria mg/day } \\
\text { Median (IQR) }\end{array}$ & $\begin{array}{l}171 \\
(114-380)\end{array}$ & $\begin{array}{l}197 \\
(114-571)\end{array}$ & $\begin{array}{l}157 \\
(111-237)\end{array}$ & $<0.05$ \\
\hline Albuminuria $\geq 30 \mathrm{mg} / \mathrm{g}$ creatinine & 109 (31.7) & $65(31.9)$ & $44(42.1)$ & ns \\
\hline Proteinuria $\geq 150 \mathrm{mg} / \mathrm{g}$ creatinine & $109(31.7)$ & $72(35.3)$ & $37(26.4)$ & ns \\
\hline $\begin{array}{l}\text { Logarithm of NT-PRO-BNP } \\
\text { Mean value + S.D. }\end{array}$ & $5.74+1.41$ & $5.90+1.47$ & $5.51+1.28$ & ns \\
\hline Cyclosporine n (\%) & $73(21.2)$ & $56(27.5)$ & $17(12.1)$ & $<0.05$ \\
\hline Tacrolimus n (\%) & $213(61.9)$ & $108(52.9)$ & $105(75.0)$ & $<0.05$ \\
\hline Steroids n (\%) & $176(51.2)$ & $92(45.1)$ & $84(60.0)$ & $<0.05$ \\
\hline
\end{tabular}




\begin{tabular}{|c|c|c|c|c|}
\hline MMF n (\%) & $150(43.6)$ & $82(40.2)$ & $68(48.6)$ & ns \\
\hline MPS n (\%) & $172(50)$ & $108(52.9)$ & $64(45.7)$ & ns \\
\hline AZA n (\%) & $5(1.5)$ & $2(1.0)$ & $3(2.1)$ & ns \\
\hline mTOR n (\%) & $10(2.9)$ & $6(2.9)$ & $4(2.9)$ & ns \\
\hline Belatacept n (\%) & $41(11.9)$ & $28(13.7)$ & $13(9.3)$ & ns \\
\hline Calcium channel blockers n (\%) & $158(45.9)$ & $106(52.0)$ & $52(37.1)$ & $<0.05$ \\
\hline Beta blockers n (\%) & $242(70.3)$ & $146(71.6)$ & $96(68.6)$ & ns \\
\hline Diuretics n (\%) & $120(34.9)$ & $86(42.2)$ & $34(24.3)$ & $<0.05$ \\
\hline EPO n (\%) & $67(19.5)$ & $56(27.4)$ & $21(15)$ & 0.07 \\
\hline
\end{tabular}

Abbreviations: RAAS, renin angiotensin aldosteron system; RTR, renal transplant recipient; BMI- body mass index; CAD, coronary artery disease; RRT, renal replacement therapy; POAD, peripheral obliterans artery disease; ESRD, end stage renal disease; KTx, kidney transplantation; NODAT, new onset diabetes after transplantation; MMF, mycophenolate mofetil; MPS, mycophenolate sodium; AZA, azathioprine; EPO, erythropoietin; SD, standard deviation; IQR, interquartile range

tionally, systolic blood pressure, diastolic blood pressure and all arterial stiffness parameters (baPWV, cfPWV, pulse pressure) did not differ significantly between the RAAS $(+)$ and RAAS $(-)$ groups (Table 2).

Among the study population, there were $38 \%$ of RTRs with cfPWV higher than $8.1 \mathrm{~m} / \mathrm{s}$. There were $39.7 \%$ and $35 \%$ of patients in the $\operatorname{RAAS}(+)$ and $\operatorname{RAAS}(-)$ groups, respectively.

\section{Arterial stiffness parameters and CVD, heart failure and diabetes}

Univariate and multivariate analysis

The results revealed that the presence of CVD in patients was associated with a significant increase in both, the PWV and pulse pressure, in univariate and multivariate analysis (Table $3 \mathrm{a}, \mathrm{b}$ ). There was no influence of diabetes, heart failure, proteinuria, albuminuria, renal function (creatinine level, eGFR), time of RRT and time after transplantation on the pulse pressure values (Table 3a). On the other hand, diabetes, heart failure, and eGFR significantly affected the pulse wave velocity. There was no influence of the creatinine level, albuminuria, and proteinuria on the pulse wave velocity in the study population (Table $3 \mathrm{~b}$ ). Both analyses showed the positive effect of beta-blockers and diuretics on arterial stiffness parameters. In the contrast, calcium channel blockers decreased the pulse pressure, but there was no effect on the pulse wave velocity. Moreover, erythropoietin administration did not influence the arterial stiffness parameters in analyzed RTRs (Table $3 a, b)$.

Table 2. Blood pressure and arterial and stiffness parameters.

\begin{tabular}{|c|c|c|c|c|}
\hline & Total study population & RAAS (+) & RAAS (-) & $\begin{array}{l}\text { P } \\
\text { RAAS+ vs RAAS- }\end{array}$ \\
\hline n (\%) & 344 & $204(59.3)$ & $140(40.7)$ & $<0.05$ \\
\hline $\begin{array}{l}\text { Systolic blood pressure right arm }(\mathrm{mmHg}) \\
\text { Mean value }+ \text { S.D. }\end{array}$ & $140.7+17.7$ & $141.1+17.5$ & $139.9+18.0$ & ns \\
\hline $\begin{array}{l}\text { Systolic blood pressure } \\
\text { left arm (mmHg) } \\
\text { Mean value + S.D. }\end{array}$ & $139.6+20.1$ & $140.2+20.3$ & $138.9+19.9$ & ns \\
\hline $\begin{array}{l}\text { Diastolic blood pressure right arm }(\mathrm{mmHg}) \\
\text { Mean value }+ \text { S.D. }\end{array}$ & $85.8+10.3$ & $85.8+10.6$ & $85.8+9.7$ & ns \\
\hline $\begin{array}{l}\text { Diastolic blood pressure left arm }(\mathrm{mmHg}) \\
\text { Mean value }+ \text { S.D. }\end{array}$ & $85.7+10.9$ & $85.7+11.9$ & $85.6+9.3$ & ns \\
\hline $\begin{array}{l}\text { Pulse pressure } \\
\text { right arm }(\mathrm{mmHg}) \\
\text { Mean value }+ \text { S.D. }\end{array}$ & $54.6+14.7$ & $54.9+15.3$ & $54.0+13.8$ & ns \\
\hline $\begin{array}{l}\text { Pulse pressure } \\
\text { left arm }(\mathrm{mmHg}) \\
\text { Mean value + S.D. }\end{array}$ & $54.0+15.9$ & $54.5+16.3$ & $53.3+15.3$ & ns \\
\hline $\begin{array}{l}\text { ba PWV right }(\mathrm{m} / \mathrm{s}) \\
\text { Median }(\mathrm{IQR})\end{array}$ & $\begin{array}{l}11.8 \\
(10.7-13.3)\end{array}$ & $\begin{array}{l}11.9 \\
(11.0-14.0)\end{array}$ & $\begin{array}{l}11.7 \\
(10.5-12.8)\end{array}$ & ns \\
\hline $\begin{array}{l}\text { ba PWV left }(\mathrm{m} / \mathrm{s}) \\
\text { Median (IQR) }\end{array}$ & $\begin{array}{l}12.0 \\
(10.9-13.5)\end{array}$ & $\begin{array}{l}11.9 \\
(10.9-13.8)\end{array}$ & $\begin{array}{l}12.1 \\
(10.6-13.2)\end{array}$ & ns \\
\hline $\begin{array}{l}\text { cf PWV (m/s) } \\
\text { Median (IQR) }\end{array}$ & $\begin{array}{l}7.9 \\
(6.9-9.5)\end{array}$ & $\begin{array}{l}8 \\
(6.9-10.1)\end{array}$ & $\begin{array}{l}7.8 \\
(6.6-8.7)\end{array}$ & $<0.05$ \\
\hline
\end{tabular}

Abbreviation: $\mathrm{ABI}$, ankle brachial index; $\mathrm{PWV}$, pulse wave velocity; $\mathrm{SD}$, standard deviation; IQR, interquartile range 
Table 3. Linear regression results for arterial stiffness parameters: pulse pressure (a) and pulse wave velocity (b).

\begin{tabular}{|c|c|c|c|c|c|c|c|c|}
\hline \multirow{2}{*}{$\begin{array}{l}\text { (a) } \\
\text { Parameter }\end{array}$} & \multicolumn{2}{|c|}{ Univariate analysis } & \multicolumn{2}{|c|}{ Multivariate analysis } & \multicolumn{2}{|c|}{ Univariate analysis } & \multicolumn{2}{|c|}{ Multivariate analysis } \\
\hline & \multicolumn{4}{|c|}{ Pulse pressure right } & \multicolumn{4}{|c|}{ Pulse pressure left } \\
\hline \multicolumn{9}{|l|}{ Model 1} \\
\hline & Coefficient & $p$ & Coefficient & $p$ & Coefficient & $p$ & Coefficient & $p$ \\
\hline CVD & 5.38 & $<0.0001$ & 5.29 & $<0.001$ & 6.73 & $<0.001$ & 4.40 & $<0.05$ \\
\hline DM & 0.09 & 0.95 & 0.11 & 0.94 & 0.66 & 0.65 & 0.39 & 0.81 \\
\hline $\mathrm{HF}$ & 0.12 & 0.91 & 0.63 & 0.59 & 1.22 & 0.59 & 0.02 & 0.98 \\
\hline \multicolumn{9}{|l|}{ Model 2} \\
\hline creatinine level mg/dl & 2.51 & 0.05 & 1.25 & 0.75 & 1.55 & 0.25 & 4.40 & 0.30 \\
\hline eGFR CKD-Epi & -0.10 & 0.05 & -0.15 & 0.22 & -0.05 & 0.15 & -0.21 & 0.13 \\
\hline Albuminuria mg/day & 0.0007 & 0.79 & 0.006 & 0.67 & 0.001 & 0.64 & 0.009 & 0.54 \\
\hline $\begin{array}{l}\text { Proteinuria } \\
\mathrm{mg} / \text { day }\end{array}$ & 0.0001 & 0.57 & 0.005 & 0.65 & 0.0003 & 0.34 & 0.008 & 0.51 \\
\hline \multicolumn{9}{|l|}{ Model 3} \\
\hline Time RRT & 0.03 & 0.06 & 0.04 & $<0.05$ & 0.02 & 0.25 & 0.02 & 0.22 \\
\hline Time after KTx & 0.02 & 0.05 & 0.02 & 0.05 & 0.02 & 0.18 & 0.01 & 0.20 \\
\hline \multicolumn{9}{|l|}{ Model 4} \\
\hline Beta blockers & -3.41 & $<0.001$ & -2.72 & $<0.001$ & -3.02 & $<0.005$ & -2.13 & $<0.05$ \\
\hline Calcium channel blockers & -3.11 & $<0.001$ & -2.61 & $<0.001$ & -3.79 & $<0.001$ & -3.39 & $<0.001$ \\
\hline diuretics & -2.15 & $<0.05$ & -0.95 & 0.38 & -2.40 & $<0.05$ & -1.26 & 0.26 \\
\hline EPO & 0.16 & 0.90 & 0.24 & 0.69 & 0.17 & 0.90 & 0.54 & 0.29 \\
\hline
\end{tabular}

\begin{tabular}{|c|c|c|c|c|c|c|c|c|c|c|c|c|}
\hline (b) & \multicolumn{2}{|c|}{ Univariate analysis } & \multicolumn{2}{|c|}{$\begin{array}{l}\text { Multivariate } \\
\text { analysis }\end{array}$} & \multicolumn{2}{|c|}{$\begin{array}{l}\text { Univariate } \\
\text { analysis }\end{array}$} & \multicolumn{2}{|c|}{$\begin{array}{l}\text { Multivariate ana- } \\
\text { lysis }\end{array}$} & \multicolumn{2}{|c|}{$\begin{array}{l}\text { Univariate ana- } \\
\text { lysis }\end{array}$} & \multicolumn{2}{|c|}{$\begin{array}{l}\text { Multivariate ana- } \\
\text { lysis }\end{array}$} \\
\hline Parameter & baPWV righ & & & & baPWV & left & & & cfPWV & & & \\
\hline \multicolumn{13}{|l|}{ Model 1} \\
\hline & Coefficient & $p$ & $\begin{array}{l}\text { Coeffi- } \\
\text { cient }\end{array}$ & $p$ & $\begin{array}{l}\text { Coeffi- } \\
\text { cient }\end{array}$ & $p$ & $\begin{array}{l}\text { Coeffi- } \\
\text { cient }\end{array}$ & $p$ & $\begin{array}{l}\text { Coeffi- } \\
\text { cient }\end{array}$ & $p$ & $\begin{array}{l}\text { Coeffi- } \\
\text { cient }\end{array}$ & $p$ \\
\hline CVD & 1.18 & $<0.001$ & 1.14 & $<0.0001$ & 1.05 & $<0.001$ & 1.03 & $<0.0001$ & 1.19 & $<0.001$ & 0.96 & $<0.0001$ \\
\hline $\mathrm{DM}$ & 0.64 & $<0.05$ & 0.52 & $<0.05$ & 0.65 & $<0.05$ & 0.52 & $<0.05$ & 0.57 & $<0.05$ & 0.45 & $<0.05$ \\
\hline $\mathrm{HF}$ & 0.50 & $<0.005$ & 0.28 & 0.09 & 0.55 & $<0.005$ & 0.35 & 0.06 & 0.49 & $<0.005$ & 0.31 & 0.06 \\
\hline \multicolumn{13}{|l|}{ Model 2} \\
\hline $\begin{array}{l}\text { creatinine } \\
\text { level mg/ } \\
\text { dl }\end{array}$ & 0.21 & 0.30 & 1.07 & 0.06 & 0.14 & 0.49 & 0.98 & 0.12 & 0.13 & 0.46 & 1.01 & 0.06 \\
\hline $\begin{array}{l}\text { eGFR } \\
\text { CKD-Epi }\end{array}$ & -0.03 & $<0.001$ & -0.07 & $<0.001$ & -0.02 & $<0.001$ & -3.11 & $<0.005$ & -0.03 & $<0.001$ & -0.06 & $<0.005$ \\
\hline $\begin{array}{l}\text { Albumi- } \\
\text { nuria mg/ } \\
\text { day }\end{array}$ & 0.0002 & 0.61 & 0.002 & 0.89 & 0.0004 & 0.35 & 0.0004 & 0.85 & 0.0003 & 0.41 & 0.0008 & 0.97 \\
\hline
\end{tabular}




\begin{tabular}{|c|c|c|c|c|c|c|c|c|c|c|c|c|}
\hline $\begin{array}{l}\text { Proteinuria } \\
\text { mg/day }\end{array}$ & 0.0002 & 0.57 & 0.002 & 0.69 & 0.0003 & 0.34 & 0.0002 & 0.92 & 0.0003 & 0.37 & 0.0005 & 0.75 \\
\hline \multicolumn{13}{|l|}{ Model 3} \\
\hline Time RRT & 0.005 & 0.08 & 0.005 & 0.05 & 0.003 & 0.25 & 0.004 & 0.21 & 0.004 & 0.10 & 0.005 & 0.07 \\
\hline $\begin{array}{l}\text { Time after } \\
\text { KTx }\end{array}$ & 0.0001 & 0.94 & 0.0005 & 0.78 & 0.0001 & 0.91 & 0.0002 & 0.93 & 0.02 & 0.98 & 0.0002 & 0.89 \\
\hline \multicolumn{13}{|l|}{ Model 4} \\
\hline $\begin{array}{l}\text { Beta bloc- } \\
\text { kers }\end{array}$ & -0.56 & $<0.001$ & -0.43 & $<0.005$ & -0.69 & $<0.0001$ & -0.52 & $<0.005$ & -0.57 & $<0.0001$ & -0.44 & $<0.005$ \\
\hline $\begin{array}{l}\text { Calcium } \\
\text { channel } \\
\text { blockers }\end{array}$ & -0.13 & 0.39 & -0.002 & 0.98 & -0.12 & 0.46 & -0.06 & 0.67 & -0.09 & 0.50 & -0.05 & 0.71 \\
\hline diuretics & -0.71 & $<0.0001$ & -0.59 & $<0.005$ & -0.91 & $<0.0001$ & -0.76 & $<0.0001$ & -0.76 & $<0.0001$ & -0.64 & $<0.0001$ \\
\hline EPO & 0.23 & 0.27 & 0.29 & 0.13 & 0.17 & 0.42 & 0.26 & 0.19 & 0.21 & 0.26 & 0.28 & 0.10 \\
\hline
\end{tabular}

Abbreviations: CVD, cardiovascular disease; eGFR, estimated glomerular filtration rate; HF, heart failure; RRT, renal replacement therapy; KTx, kidney transplantation; DM, diabetes; EPO, erythropoietin; baPWV, brachial ankle pulse wave velocity; cfPWV, carotid femoral pulse wave velocity

Table 4. Blood pressure and arterial stiffness parameters in CVD, HF and Diabetes patients with or without RAAS blockers.

\begin{tabular}{|c|c|c|c|c|}
\hline & Total study population & RAAS (+) & RAAS (-) & $\begin{array}{l}\text { P } \\
\text { RAAS+ vs RAAS- }\end{array}$ \\
\hline $\begin{array}{l}\mathrm{n}(\%) \\
\operatorname{CVD}(+)\end{array}$ & 77 & $57(74)$ & $20(26)$ & $<0.05$ \\
\hline $\begin{array}{l}\text { Systolic blood pressure right arm }(\mathrm{mmHg}) \\
\text { Mean value + S.D. }\end{array}$ & $145.5+18.6$ & $145.6+18.6$ & $145.1+19.6$ & ns \\
\hline $\begin{array}{l}\text { Systolic blood pressure } \\
\text { left arm (mmHg) } \\
\text { Mean value + S.D. }\end{array}$ & $148.4+26.1$ & $148.4+28.0$ & $148.6+21.4$ & ns \\
\hline $\begin{array}{l}\text { Diastolic blood pressure right arm }(\mathrm{mmHg}) \\
\text { Mean value + S.D. }\end{array}$ & $81.4+10.4$ & $80.9+10.1$ & $83+11.6$ & ns \\
\hline $\begin{array}{l}\text { Diastolic blood pressure left arm }(\mathrm{mmHg}) \\
\text { Mean value + S.D. }\end{array}$ & $83.1+11.8$ & $82.7+12.5$ & $84.2+10$ & ns \\
\hline $\begin{array}{l}\text { Pulse pressure } \\
\text { right arm }(\mathrm{mmHg}) \\
\text { Mean value + S.D. }\end{array}$ & $63.2+17.5$ & $63.5+17.6$ & $62.1+18$ & ns \\
\hline $\begin{array}{l}\text { Pulse pressure } \\
\text { left arm (mmHg) } \\
\text { Mean value + S.D. }\end{array}$ & $65.3+21.4$ & $65.7+22$ & $64.4+20.9$ & ns \\
\hline $\begin{array}{l}\text { ba PWV right }(\mathrm{m} / \mathrm{s}) \\
\text { Median (IQR) }\end{array}$ & $\begin{array}{l}14.7 \\
(12.7-15.9)\end{array}$ & $\begin{array}{l}15 \\
(12.7-16)\end{array}$ & $\begin{array}{l}12.9 \\
(12.4-15.3)\end{array}$ & ns \\
\hline $\begin{array}{l}\text { ba PWV left (m/s) } \\
\text { Median (IQR) }\end{array}$ & $\begin{array}{l}14.1 \\
(12.6-16,0)\end{array}$ & $\begin{array}{l}14.4 \\
(12.6-16.3)\end{array}$ & $\begin{array}{l}13.2 \\
(13-15.8)\end{array}$ & ns \\
\hline $\begin{array}{l}\text { cf PWV (m/s) } \\
\text { Median (IQR) }\end{array}$ & $\begin{array}{l}10.5 \\
(8.45-11.9)\end{array}$ & $\begin{array}{l}10.6 \\
(8.7-12.2)\end{array}$ & $\begin{array}{l}8.6 \\
(8-10.8)\end{array}$ & ns \\
\hline $\begin{array}{l}\mathrm{n}(\%) \\
\text { Diabetes }(+)\end{array}$ & 65 & $41(63.1)$ & $24(36.9)$ & $<0.05$ \\
\hline $\begin{array}{l}\text { Systolic blood pressure right arm }(\mathrm{mmHg}) \\
\text { Mean value }+ \text { S.D. }\end{array}$ & $143.9+18.6$ & $145.9+18.8$ & $140.4+18.3$ & ns \\
\hline $\begin{array}{l}\text { Systolic blood pressure } \\
\text { left arm (mmHg) } \\
\text { Mean value + S.D. }\end{array}$ & $144.9+24.2$ & $149.5+25.8$ & $138.7+21.1$ & ns \\
\hline $\begin{array}{l}\text { Diastolic blood pressure right arm }(\mathrm{mmHg}) \\
\text { Mean value }+ \text { S.D. }\end{array}$ & $84.5+8.6$ & $83.7+8.4$ & $85.9+8.8$ & ns \\
\hline $\begin{array}{l}\text { Diastolic blood pressure left arm }(\mathrm{mmHg}) \\
\text { Mean value + S.D. }\end{array}$ & $84.6+11.4$ & $83.9+10.9$ & $85.5+12.2$ & ns \\
\hline $\begin{array}{l}\text { Pulse pressure } \\
\text { right arm }(\mathrm{mmHg}) \\
\text { Mean value + S.D. }\end{array}$ & $59.4+16.9$ & $62.1+18.4$ & $54.5+13.1$ & ns \\
\hline
\end{tabular}




\begin{tabular}{|c|c|c|c|c|}
\hline $\begin{array}{l}\text { Pulse pressure } \\
\text { left arm (mmHg) } \\
\text { Mean value + S.D. }\end{array}$ & $60.3+19.5$ & $65.6+22.1$ & $53.2+12.5$ & $<0.05$ \\
\hline $\begin{array}{l}\text { ba PWV right }(\mathrm{m} / \mathrm{s}) \\
\text { Median }(\mathrm{IQR})\end{array}$ & $\begin{array}{l}12.9 \\
(11.6-14.8)\end{array}$ & $\begin{array}{l}13.3 \\
(12.4-15.5)\end{array}$ & $\begin{array}{l}12.5 \\
(11.2-14.2)\end{array}$ & ns \\
\hline $\begin{array}{l}\text { ba PWV left }(\mathrm{m} / \mathrm{s}) \\
\text { Median (IQR) }\end{array}$ & $\begin{array}{l}13.8 \\
(12.1-16.3)\end{array}$ & $\begin{array}{l}14.3 \\
(12-17)\end{array}$ & $\begin{array}{l}13.2 \\
(12.1-15.2)\end{array}$ & $\mathrm{ns}$ \\
\hline $\begin{array}{l}\text { cf PWV (m/s) } \\
\text { Median (IQR) }\end{array}$ & $\begin{array}{l}8,7 \\
(7,4-11,2)\end{array}$ & $\begin{array}{l}9,1 \\
(8-11,6)\end{array}$ & $\begin{array}{l}8,6 \\
(7,3-9,9)\end{array}$ & ns \\
\hline $\begin{array}{l}\text { n }(\%) \\
\text { Heart failure }(+)\end{array}$ & 90 & $56(62.2)$ & $34(37.8)$ & $<0.05$ \\
\hline $\begin{array}{l}\text { Systolic blood pressure right arm }(\mathrm{mmHg}) \\
\text { Mean value }+ \text { S.D. }\end{array}$ & $140.8+17.6$ & $142.6+18.6$ & $137.6+15.3$ & ns \\
\hline $\begin{array}{l}\text { Systolic blood pressure } \\
\text { left arm }(\mathrm{mmHg}) \\
\text { Mean value }+ \text { S.D. }\end{array}$ & $140.8+18.7$ & $140.1+20.0$ & $142+16.8$ & ns \\
\hline $\begin{array}{l}\text { Diastolic blood pressure right arm }(\mathrm{mmHg}) \\
\text { Mean value + S.D. }\end{array}$ & $83.7+10.0$ & $82.4+11.13$ & $86.2+7.1$ & ns \\
\hline $\begin{array}{l}\text { Diastolic blood pressure left arm }(\mathrm{mmHg}) \\
\text { Mean value + S.D. }\end{array}$ & $84.3+11.4$ & $82.0+12.8$ & $88.3+7.22$ & $<0.05$ \\
\hline $\begin{array}{l}\text { Pulse pressure } \\
\text { right arm }(\mathrm{mmHg}) \\
\text { Mean value }+ \text { S.D. }\end{array}$ & $56.9+17.2$ & $60.1+18.9$ & $51.1+11.9$ & $<0.05$ \\
\hline $\begin{array}{l}\text { Pulse pressure } \\
\text { left arm (mmHg) } \\
\text { Mean value + S.D. }\end{array}$ & $56.5+15.9$ & $58.2+17.1$ & $53.7+13.6$ & ns \\
\hline $\begin{array}{l}\text { ba PWV right }(\mathrm{m} / \mathrm{s}) \\
\text { Median }(\mathrm{IQR})\end{array}$ & $\begin{array}{l}12.6 \\
(11-14.5)\end{array}$ & $\begin{array}{l}12.7 \\
(11.4-15.4)\end{array}$ & $\begin{array}{l}12.3 \\
(10.7-14.1)\end{array}$ & ns \\
\hline $\begin{array}{l}\text { ba PWV left }(\mathrm{m} / \mathrm{s}) \\
\text { Median }(\mathrm{IQR})\end{array}$ & $\begin{array}{l}12.7 \\
(11.3-14.9)\end{array}$ & $\begin{array}{l}12.7 \\
(11.1-14.6)\end{array}$ & $\begin{array}{l}12.8 \\
(11.6-15.2)\end{array}$ & ns \\
\hline $\begin{array}{l}\text { cf PWV (m/s) } \\
\text { Median (IQR) }\end{array}$ & $\begin{array}{l}8.4 \\
(7.2-10.7)\end{array}$ & $\begin{array}{l}8.4 \\
(7.2-11.6)\end{array}$ & $\begin{array}{l}8.1 \\
(7-10.4)\end{array}$ & ns \\
\hline
\end{tabular}

Abbreviations: CVD,cardiovascular disease; RTR, renal transplant recipient; HF, heart failure; baPWV, brachial ankle pulse wave velocity; cfPWV, carotid femoral pulse wave velocity; + S.D., standard deviation; IQR, interquartile range

No difference was observed in SBP, DBP, and arterial stiffness between patients with CVD, diabetes, and HF treated with RAAS and not treated with RAAS (Table 4).

\section{DISCUSSION}

Our study showed an indication bias of the RAAS prescription, and no conclusion on the influence of RAAS on arterial stiffness can be drawn. Moreover, in patients with CVD, heart failure and diabetes, higher arterial stiffness was observed in comparison to participants without these comorbidities. Additionally, a worse renal graft function correlated with arterial stiffness. Furthermore, the usage of RAAS blockade in participants with CVD, heart failure and diabetes did not cause a difference in terms of the value of PWV.

It is well known that the RAAS blockade ameliorates hypertension and proteinuria which influence the renal graft-survival (Tylicki et al., 2007; Sennesael et al., 1995). Additionally, Szabo and others (Szabo et al., 2000) showed that ACEIs and ARBs may protect the renal graft from fibrosis and tubular atrophy.

On the other hand, Suwelack et al. observed that the left ventricle mass index and diastolic relaxation improved after two years of treatment with quinapril, when compared to atenolol after KTx (Suwelack et al., 2000). Nevertheless, there is no sufficient data regarding the influence of the RAAS blockade on arterial stiffness in RTRs. In the study presented here, we showed a more advanced arterial stiffness and a higher level of creatinine in the RAAS $(+)$ when compared to RAAS $(-)$ patients. It is worth to underline that the prevalence of CVD, heart failure and diabetes was higher in the former population. Moreover, all of these comorbidities correlated with arterial stiffness parameters, but CVD had the strongest impact.

Kolonko and others (Kolonko et al., 2016) reported that pre-transplantation diabetes and CVD significantly correlated with an increased PWV in RTRs. Moreover, Kim and others (Kim et al., 2015) analyzed baPWV in the end-stage renal disease patients that were on the waiting list for kidney transplantation, and showed a higher baPWV in patients with presence of CVD in their medical history than in those without them. The authors proved that PWV was a strong predictor of CVD in RTRs. Another study also showed that PWV was associated with CVD events after KTx. Additionally, pulsatile stress was a significant (HR 3.7; $p<0.02)$ and independent factor for cardiovascular events in RTRs, in addition to a past history of cardiovascular events (HR 1.16; $p<0.04$ ) (Bahous et al., 2004). Therefore, CVD and arterial stiffness are interrelated, as it was observed in our study.

Similar results were found in a Belgian study. After a mean follow-up of 5 years, Verbeke and others (Verbeke et al., 2011) noted in a cohort of 512 RTRs that cfPWV was a significant factor in the assessment of a cardiovascular risk. Moreover, patients with a cfPWV of $\geq 8.1 \mathrm{~m} / \mathrm{s}$ had worse cardiovascular survival when com- 
pared to patients with a cfPWV $<8.1 \mathrm{~m} / \mathrm{s}$. In our study, there were up to $38 \%$ patients with cfPWV $>8.1 \mathrm{~m} / \mathrm{s}$, which qualified them to a higher cardiovascular risk, regardless of comorbidities, age and immunosuppressive regimen.

Ayub and others (Ayub et al., 2015) performed the PWV measurement and estimation of the estimated glomerular filtration rate in RTRs and showed an inverse correlation between these two parameters. Moreover, the authors suggested the necessity of PWV evaluation in the assessment of cardiovascular risk in RTRs with worse renal graft function. Similarly, the results of a study from Turkey showed a significantly higher value of PWV in RTRs with lower estimated glomerular filtration rate. The mean value of PWV was $7.4 \pm 0.6$ vs $6.1 \pm 0.4 \mathrm{~cm} / \mathrm{s}$ in patients with estimated glomerular filtration rate $15-49 \mathrm{ml} / \mathrm{min}$ and $50-69 \mathrm{ml} / \mathrm{min}$, respectively (Sezera et al., 2015). Likewise, in our study, there was a significant correlation between arterial stiffness and renal graft function, assessed by glomerular filtration rate.

Proteinuria is acknowledged as a marker of renal graft damage, a predictor of graft survival, and the incidence of CVD in RTRs. Its prevalence was described in up to $45 \%$ of RTRs (Park et al., 2000; Amer et al., 2007). The prevalence of proteinuria in our cohort was $31.7 \%$, which is consistent with the above data.

Guliyev and others (Guliyev et al., 2015) showed that proteinuria (>500 mg/day) was strongly associated with increased risk of cardiovascular events, according to accelerated arterial stiffness and decreased arterial elasticity when compared to patients with lower proteinuria $(<500$ $\mathrm{mg} /$ day).

Among RTRs in Germany, Baumann et al. reported that pulsatile stress, not PWV was associated with the quantity of albuminuria $(\mathrm{r}=0.29 ; p<0.01$ and $\mathrm{r}=0.06$; $p=0.6$ respectively). Therefore, this parameter could be a marker for arterial dysfunction in RTRs (Baumann et al., 2010). Jeon and others (Jeon et al., 2015) evaluated the incidence of major adverse cardiac events (cardiac death, nonfatal myocardial infarction, or coronary revascularization) within 55.3months of follow-up. They showed that proteinuria was associated with major adverse cardiac events (hazard ratio [HR] 8.689, 95\% confidence interval $[\mathrm{CT}] 2.929-25.774, p<0.001)$ when compared to those without proteinuria. The mortality rate among the study population was significantly higher in patients with proteinuria (HR 6.815, 95\% CI 2.164-21.467, $p=0.001$ ).

In our study, proteinuria and albuminuria are not correlated with PWV.

Aspirin, beta-blockers, ACEIs, ARBs, and statins can each reduce the risk of major CVD events by $25 \%$, in the general population (Yusuf et al., 2002). However, there are no sufficient data regarding the safety and efficacy of ACEis or ARBs treatments in reducing cardiovascular risk in RTRs.

Gastona and others (Gastona et al., 2009) analyzed the use of cardioprotective medications in RTRs based on The Long Term Deterioration of Kidney Allograft Function (DeKAF) study.

The results indicated that the RAAS blockade was used in $24 \%$ of the study population at 6 months after transplantation and the authors presented skepticism concerning the prescription of CVD medication in that population (Gastona et al., 2009).

On the other hand, Pilmore and others (Pilmore et al., 2011) reported that the prevalence of ACEIs or ARBs administration in RTRs, 5 years after transplantation, was at $36.3 \%$. Patients with the history of myocardial infarc- tion used these agents more frequently. In comparison, administration of the RAAS blockade did not differentiate between RTRs with and without diabetes.

Our data showed a higher prevalence of ACEIs or ARBs administration in RTRs as compared to the cited article. Moreover, a majority of participants with CVD or diabetes have used these medications. This demonstrated the high awareness of the transplant center about the possible role of the RAAS blockade in reducing the cardiovascular risk in that population.

A small reduction in pulse wave velocity was observed in the ZEUS study, under zofenopril or irbesartan treatment (Omboni et al., 2017). Zhao and others (Zhao et al., 2018) using the rat kidney transplantation model, showed a significant mitigation of the angiotensin IIinduced contractions in the aorta and mesenteric arteries of the recipient under losartan treatment.

There are no sufficient data concerning the influence of the RAAS blockade on arterial stiffness in RTRs as compared to the chronic kidney disease population. The efficacy and safety treatment with enalapril and/or candesartan was shown by Frimodt-Møller et al., among patients with mean GFR 30, range 13-59 ml/min/1.73 $\mathrm{m}^{2}$. The authors showed a decrease in PWV during the 24 weeks of follow-up in the analyzed population (FrimodtMøller et al., 2012).

Our study did not find a difference in PWV in high risk (CVD, diabetes, heart failure) RTRs who were treated with the RAAS blockade in comparison to participants without these medications. The results are not consistent with the above data. There is association with bias. Additionally, our study population was heterogeneous in terms of different time after transplantation and the time of RAAS blockers administration, and was not adjusted for these parameters.

\section{LIMITATIONS}

There are several limitations of this study that should be considered when interpreting our conclusions. First of all, this is a single-center study. The sample size was relatively small, but to the best of our knowledge, this is the first study where the influence of the RAAS blockade on arterial stiffness was analyzed in RTRs. Moreover, the amount of patients differed in the RAAS $(+)$ and RAAS $(-)$ groups. Secondly, the study population was a heterogeneous group, with different comorbidities, including cardiovascular disease, diabetes and heart failure, dissimilar time of dialysis, various periods after renal transplantation and the RAAS blockade treatment. Additionally, there were discrepancies in terms of the eGFR, proteinuria, and albuminuria values, and immunosuppressive regimen between the RAAS $(+)$ and RAAS $(-)$ patients. Thirdly, there was no follow-up in the study. Fourthly, the doses of ARBs and ACEIs used in the study population were small. Therefore, the influence of these agents on arterial stiffness parameters was non-significant.

However, despite these limitations, the study highlights some important information for the RTRs in terms of prevention of CVD in this population. Hence, we cannot exclude that there is no effect of the RAAS blockade on arterial stiffness at all. Furthermore, we cannot exclude the possibility of residual confounding. To obtain a definite answer on the effect of RAAS blockade on PWV, further studies are needed. 


\section{CONCLUSIONS}

The study showed an indication bias of the RAAS prescription. Moreover, no conclusion on the influence of RAAS on arterial stiffness can be drawn. On the other hand, the results indicated diuretics and beta-blockers as agents lowering the arterial stiffness in RTRs.

There is a necessity for further evaluation of the nephroprotective role of the RAAS blockade in randomized clinical trials in the RTRs population.

\section{Ethical approval and consent to participate}

This study was approved by The Ethics Committee of Charité - Universitätsmedizin Berlin (EA 1/252/17)

On behalf of all authors, the corresponding author states that there is no conflict of interest. All procedures performed in studies involving human participants were under the ethical standards of the institutional and/or national research committee and with the 1964 Helsinki declaration and its later amendments or comparable ethical standards. Informed consent was obtained from all individual participants included in the study.

\section{Competing interests}

The authors declare that they have no competing interests.

\section{Authors' contributions}

Substantial contributions to the conception or design of the work; or acquisition, analysis, or interpretation of data for the work: ZH, SI, PB, SB, ADŚ, KB, FH.

Drafting the work or revising it critically for important intellectual content: $\mathrm{ZH}, \mathrm{PB}, \mathrm{ADS}, \mathrm{KB}$. $\mathrm{KB}$.

Final approval of the version to be published: ADŚ,

Agreement to be accountable for all aspects of the work in ensuring that questions related to the accuracy or integrity of any part of the work are appropriately investigated and resolved: $\mathrm{ZH}, \mathrm{ADS}, \mathrm{KB}, \mathrm{FH}$. All authors have read and approved the manuscript.

\section{Availability of data and materials}

The datasets used for this study are available from the corresponding author on reasonable request.

\section{REFERENCES}

Amer H, Fidler ME, Myslak M, Morales P, Kremers WK, Larson TS, Stegall MD, Cosio MG (2007) Proteinuria after kidney transplantation, relationship to allograft histology and survival. Am. J. Transplant. 7: 2748-2756. https://doi.org/10.1111/j.16006143.2007.02006.x

Ayub M, Ullah K, Masroor I, Butt G (2015) Predictive factors for increased aortic pulse wave velocity in renal transplant recipients and its relations to graft outcome. Saudi. J. Kidney. Dis.Transplant. 26: 1130-1134. https://doi.org/10.4103/1319-2442.168581

Bahous SA, Stephan A, Barakat W, Blacher J, Asmar R, Safar ME (2004) Aortic pulse wave velocity in renal transplant patients. Kidney Int. 66: 1486-1492. https://doi.org/10.1111/j.15231755.2004.00912.x

Barenbrock M, Kosch M, Joster E, Kisters K, Rahn KH, Hausberg M (2002) Reduced arterial distensibility is a predictor of cardiovascular disease in patients after renal transplantation. J. Hypertens. 20: 7984. https://doi.org/10.1097/00004872-200201000-00012

Baumann M, Pan ChR, Roos M, von Eynatten M, Sollinger D, Lutz J, Heemann U (2010) Pulsatile stress correlates with (micro-)albuminuria in renal transplant recipients. Transpl. Int. 23: 292-298. https://doi.org/10.1111/j.1432-2277.2009.00981.x

Boutouyrie P, Fliser D, Goldsmith D, Covic A, Wiecek A, Ortiz A, Martinez-Castelao A, Lindholm B, Massy ZA, Suleymanlar G, Sicari R, Gargani L, Parati G, Mallamaci F, Zoccali C, London GM
(2015) Assessment of artery stiffness for clinical and epidemiological studies: renal and cardiovascular medicine registry. Nephrol. Dial. Transplant. 29: 232-239. https://doi.org/10.1093/ndt/gft309

Frimodt-Møller M, Kamper AL, Strandgaard S, Kreiner S, Nielsen $\mathrm{AH}$ (2012) Beneficial effects on arterial stiffness and pulse-wave reflection of combined enalapril and candesartan in chronic kidney disease - a randomized trial. PLoS One 7: e41757. https://doi. org/10.1371/journal.pone.0041757

Gastona RS, Kasiskeb BL, Fiebergc AM, Leduc R, Cosio FC, Gourishankar S, Halloran P, Hunsicker L, Rush D, Matas AJ (2009) Use of cardioprotective medications in kidney transplant recipients. Am. J. Transpl. 9: 1811-1815. https://doi.org/10.1111 /j.1600-6143.2009.02696

Guliyeva O, Sayina B, Uyara ME, Genctoy G, Sezer S, Bal Z, Demirci BG, Haberal M (2005) High-grade proteinuria as a cardiovascular risk factor in renal transplant recipients. Transplant. Proc. 47: 11701173. https://doi.org/10.1016/j.transproceed.2014.10.062

Holdaas H, de Fijter JW, Cruzado JM, Massari P, Nashan B, Kanellis J, Witzke O, Gutierrez-Dalmau A, Turkmen A, Wang Z, Lopez P, Bernhardt P, Kochuparampil J, van der Giet M, Murbraech K (2017) Cardiovascular parameters to 2 years after kidney transplantation following early switch to everolimus without calcineurin inhibitor therapy: an analysis of the randomized ELEVATE study. Transplantation 101: 2612-2620. https://doi.org/10.1097/ TP.0000000000001739

Jeon HJ, Kim CT, An JN, Lee H, Kim H, Park SK, Joo KW, Lim CS, Jung IM, Ahn C, Kim YS, Kim YH, Lee JP (2015) Time-varying maximal proteinuria correlates with adverse cardiovascular events and graft failure in kidney transplant recipients. Nephrology 20: 945951. https://doi.org/10.1111/nep.1252

Kim HS, Seung J, Lee JH, Chung BH, Yang CW (2015) Clinical significance of pre-transplant arterial stiffness and the impact of kidney transplantation on arterial stiffness. PLoS One 10: v1-v8. https:// doi.org/10.1371/journal.pone.0139138

Kolonko A, Chudek J, Szotowska M, Kuczera P, Wiecek A (2016) Cardiovascular risk factors and markers of atherosclerosis in stable kidney transplant recipients. Transplant. Proc. 48: 1543-1550. https:// doi.org/10.1016/j.transproceed.2015.12.134

Laurent S, Boutouyrie P, Asmar R, Gautier I, Laloux B, Guize L, Ducimetiere P, Benetos A (2001) Aortic stiffness is an independent predictor of all-cause and cardiovascular mortality in hypertensive patients. Hypertension 37: 1236-241. https://doi.org/10.1161/01. hyp. 37.5 .123

Laurent S, Cockcroft J, Van Bortel L, Boutouyrie P, Giannattasio C, Hayoz D, Pennier B, Vlachopoulos Ch, Wilkinson I, StruijkerBoudier H (2006) Expert consensus document on arterial stiffness: Methodological issues and clinical applications. Eur. Heart. J. 27: 2588-2605. https://doi.org/10.1093/eurheartj/ehl254

Mattace-Raso FU, van der Cammen TJ, Hofman A, van Popele NM, Bos ML, Schalekamp MA, Asmar R, Reneman RS, Hoeks APG, Breteler MMB, Witteman JCM (2006) Arterial stiffness and risk of coronary heart disease and stroke: The Rotterdam Study. Circulation 113: 657-63. https://doi.org/10.1161/01.hyp.37.5.1236

Omboni S, Malacco E, Napoli C, Modesti PA, Manolis A, Parati G, Agabiti-Rosei E, Borghi C (2017) Efficacy of zofenopril vs. irbesartan in combination with a thiazide diuretic in hypertensive patients with multiple risk factors not controlled by a previous monotherapy: a review of the double-blind, randomized " $Z$ " studies. $A d v$. Ther. 36: 784-798. https://doi.org/10.1007/s12325-017-0497-8

Park JH, Park JH, Bok HJ, Kim BS, Yang CW, Kim YS, Moon IS, Koh YB, Bang BK (2000) Persistent proteinuria as a prognostic factor for determining long-term graft survival in renal transplant recipients. Transplant. Proc. 32: 1924. hhttps://doi.org/10.1016/s00411345(00)01494-9

Pilmore HL, Skeans MA, Snyder JJ, Israni AK, Kasiske BL (2011) Cardiovascular disease medications after renal transplantation: results from the patient outcomes in renal transplantation study. Transplantation 91: 542-551. https://doi.org/10.1097/TP.0b013e31820437bd

Sennesael J, Lamote J, Violet I, Tasse S, Verbeelen D (1995) Comparison of perindopril and amlodipine in cyclosporine-treated renal allograft recipients. Hypertension 26: 436-444. https://doi. org/10.1161/01.hyp.26.3.436

Seron D, Moresco F, Grinyo JM (2001) Prevention and management of late renal allograft dysfunction. J. Nephrol. 14: 71-79.

Sezera S, Gurlek Demircia B, Guliyeva O, Sayina CB, Colaka T, Ozdemir Aca FN, Haberal M (2015) Graft function and arterial stiffness: can bioimpedance analysis be useful in renal transplant recipients? Transplant. Proc. 47: 1182-1185. https://doi.org/10.1016/j. transproceed.2014.10.067

Silvariño R, Rios P, Baldovinos G, Chichet MA, Perg N, Sola L, Soana G, De Souza N, Lamadrid V, Gadola L (2019) Is chronic kidney disease progression influenced by the type of renin-angiotensin-system blocker used? Nephron 143: 100-107. https://doi. org $/ 10.1159 / 000500925$

Suwelack B, Gerhardt U, Hausberg M, Rahn KH, Hohage H (2000) Comparison of quinapril versus atenolol: effects on blood pressure 
and cardiac mass after renal transplantation. Am. J. Cardiol. 86: 583 585. https://doi.org/10.1016/s0002-9149(00)01024-9

Szabo A, Lutz J, Schleimer K, Antus B, Hamar P, Philipp T, Heemann U (2007) Effect of angiotensin converting enzyme inhibition on growth factor mRNA in chronic renal allograft rejection in the rat. Kidney Int. 57: 982-991. https://doi.org/10.1046/j.15231755.2000.00926.x

Tylicki L, Biedunkiewicz B, Chamienia A, Wojnarowski K, Zdrojewski Z, Aleksandrowicz E, Lysiak-Szydlowska W, Rutkowski B (2007) Renal allograft protection with angiotensin II type 1 receptor antagonists. Am. J. Transplant. 7: 243-248. https://doi.org/10.1111/ j.1600-6143.2006.01588.x

Verbeke F, Marechal C, Van Laecke S, Van Biesen W, Devuyst O, Van Bortel LM, Jadoul M, Vanholder R (2011) Aortic stiffness and central wave reflections predict outcome in renal transplant recipients. Hypertension 58: 833-838. https://doi.org/10.1161/HYPERTENSIONAHA.111.176594

Vlahakos D, Marathias KP, Groyannis B, Madia N (2003) Posttransplant erythrocytosis. Kidney Int. 63: 1187-1194. https://doi. org/10.1046/j.1523-1755.2003.00850.x

Yusuf S (2002) Two decades of progress in preventing vascular disease. Lancet 360: 2-3. https://doi.org/10.1016/S0140-6736(02)09358-3

Zhao Y, Zhu Q, Sun S, Qiu Y, Li J, Liu W, Yuan G, Ma H (2018) Renal transplantation increases angiotensin II receptor-mediated vascular contractility associated with changes of epigenetic mechanisms. Int. J. Mol. Med. 41: 2375-2388. https://doi.org/10.3892/ ijmm.2018.3435 